\title{
Low-frequency electromagnetic shock machine
}

\author{
V.Yu. Neyman \\ Novosibirsk State Technical University \\ Novosibirsk, Russian Federation \\ nv.nstu@ngs.ru
}

\author{
L.A Neyman \\ Novosibirsk State Technical University \\ Novosibirsk, Russian Federation \\ neyman31@gmal.com
}

\begin{abstract}
Pulsed electromagnetic machines of shock action based on new vibro-impact technologies are widely applied in industry. Such machine striker mechanical vibrations frequency is equal to or multiple of the frequency of the supply network. The research purpose is the impact energy increase while pulse linear electromagnetic machine influence on a single-phase network power frequency is reduced. An improved version of a two-coil synchronous electromagnetic impact machine is considered, which involves dispersing the striking mass of a striker with electromagnetic forces in the forward and backward directions for a time equal to the duration of one voltage period. A new control method has been implemented to reduce the effect of the electric drive on the supply network by applying three half-wave voltages to the coils during the operating cycle time. With respect to the energy balance of the electromagnetic impact machine electromechanical system, the process of energy transformation for a full working cycle is considered, taking into account the interaction between all the elements of the impact unit. A mathematical model of the dynamics of a two-coil synchronous electromagnetic shock machine is proposed. The model is distinguished by the possibility of modeling interrelated electromechanical processes taking into account the nonlinearity of the characteristics of magnetic materials, the degree of mobility of inertial masses, the properties of elastic bonds and power losses. The computational algorithm is proposed together with an example of a numerical model in Matlab Simulink. Methods of mathematical modeling have confirmed the efficiency of the working cycle to improve the electromagnetic compatibility of the energy source with an electromagnetic shock unit.
\end{abstract}

Keywords - synchronous electromagnetic machine, impact unit, duty cycle, energy balance, mathematical model, mechanical oscillatory system, elastic connections, Lagrange equations of the second kind.

\section{INTRODUCTION}

Linear electromagnetic machines based on the impact operation principle are widely applied in practice [1-2].

Electromagnetic machines are simple devices. They have high reliability as well as the ability to reduce power consumption in resonant modes [2-4].

Despite calculating methods of electromagnetic machines are widely known, they are still improved $[4,5]$.

Special problems arise in calculation of dynamic modes, because of the peculiarities of the machines' operating cycles, their control algorithms and energy transformation processes $[4,5]$.

The impact machine impulse power consumption from a $50 \mathrm{~Hz}$ voltage source limits the single impact energy, depending on the permissible pulse power.

Therefore, the perspective direction is the creation of electromagnetic machines with high impact energy with new cycles and control methods that will ensure the reduction of the amplitude of the impact of the electric drive on the power network.

The implementation of the proposed working cycle and control method, in contrast to the known machines, allow using half-wave voltages during the working cycle. Two halfwave voltages are applied to the return coil, and the third halfwave voltage is applied to the travel coil, repeating the specified sequence.

The striker acquires the necessary kinetic energy by feeding the three impulses of voltage (current) to the coils.

The development of a dynamic model of a two-coil electromagnetic shock or impact machine should provide the capability of the comprehensive analysis of work processes in different modes.

\section{VARIANT OF LINEAR SYNCHRONOUS ELECTROMAGNETIC MACHINE}

One of the impact unit variants in the linear synchronous double-coiled electromagnetic machine with a reverse is shown in Fig. 1.

Fig. 2 provides a new operation cycle of the machine in the form of the displacement diagram, where $x$ is the head, $u$, $i$ are correspondingly the voltage and current of the operation and reverse coils, receiving power from a half-wave singlephase industrial frequency voltage source.

The impact unit (Fig. 1) consists of working tool 1, head 2, magnetic core 3 , magnetizing coil 5 , reverse coil 6 . There is the reverse device, consisting of inertial converter 7 and buffer springs 4 behind the impact unit.

To implement the operation cycle, the system of the two coils is applied. 
Impact unit operation of the electromagnetic machine consists of the following steps. When voltage pulse is applied to operating coil 5, head 2 strikes instrument 1 . After a rebound from the tool, the head accelerates in the opposite direction under the electromagnetic forces action of reverse coils 6 . Then the head impacts inertial converter 7 , which moves towards the head due to the elastic force of the buffer spring. After the impact, the head changes the direction in the opposite. Under the action of the reverse coil electromagnetic forces the head moves in the direction of the operating instrument. Next voltage impulse is applied to the operating coil 5. Under the coil electromagnetic forces action, the head moves rapidly in the direction of the operating instrument and impacts it. After being impacted by the head, the inertial

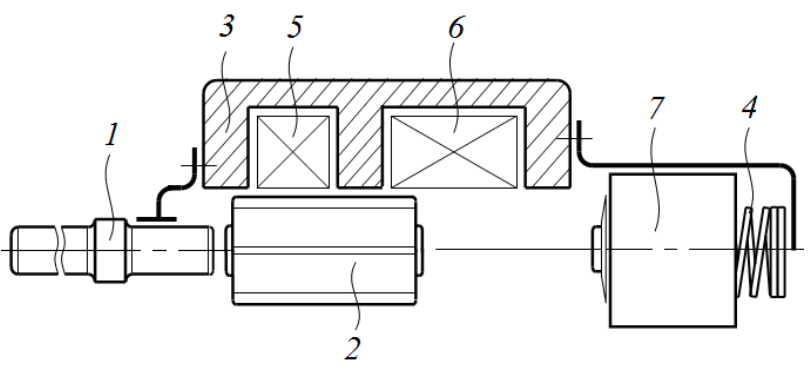

Fig. 1. Linear synchronous electromagnetic machine with inertial reversal of striker

converter moves in the direction opposite to the head motion direction and squeezes damping spring 4. Further, the cycle repeats.

The operation cycle of the impact unit is implemented with full synchronization of electric drive operation time by means of a specified algorithm.

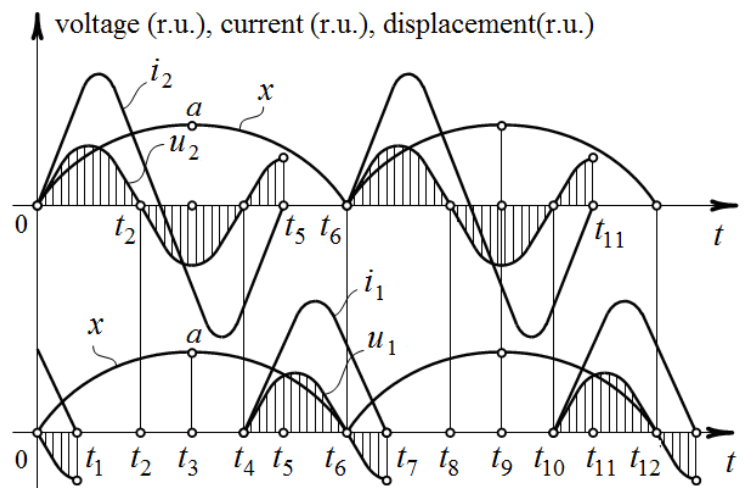

Fig. 2. New operating cycle of electromagnetic machine

Full cycle of the impact unit will exceed one period of the supply voltage source. When $f=50 \mathrm{~Hz}$, the head impact frequency $n_{\text {st }}$ will be synchronous and the operation cycle time $t_{\mathrm{c}}$ could be defined by the following expression:

$$
n_{\mathrm{st}}=\frac{60 f}{2 p}=2000 \mathrm{~min}^{-1} ; \quad t_{\mathrm{c}}=\frac{2 p}{f}=0,03 \mathrm{~s},
$$

where $2 p=1,5$ is the number of voltage periods.

\section{ENERGY CONVERSION IN OPERATING CYCLE}

The energy conversions during one working cycle are shown in the diagrams in Fig. 2.

The first phase of energy conversion begins from the point $t=0$ (Fig. 2) and coincides with the time of applying the voltage impulse to the reverse coil.

In the time interval of $0 \ldots t_{1}$ the reverse coil has a reserve of magnetic energy and it will cause the head electromagnetic braking. The operating cycle coil does not consume the electric power. Outside forces, mechanical work is spent for the head electromagnetic braking and converted into magnetic energy, with subsequent conversion into electric energy and returning to the network, while the energy of the operating coil heat loss is compensated.

The equation of the coil energy balance is:

$$
\Delta w_{\mathrm{m} 1} x, t+\int_{0}^{t_{1}} f_{\mathrm{em} 1} v d t=\int_{0}^{t_{1}} i_{1}^{2} R_{1} d t+\Delta w_{\mathrm{el} .1} x, t,
$$

where $\Delta w_{\mathrm{m} 1} \quad x, t$ is the magnetic field residual energy of the coil retained from the previous cycle; $f_{\mathrm{em} 1}$ is the coil electromagnetic force equal to the head electromagnetic braking; $v$ is the head speed, $i_{1}^{2} R_{1}$ is the power heat loss of the coil; $i_{1}, R_{1}$ are correspondingly the current and the resistance of the coil; $\Delta w_{\text {el. } 1} x, t$ is the electric power returned to the network by the operation cycle coil.

At the time interval $0 \ldots t_{2}$ (Fig. 2), head movement in the direction of the inertia converter is carried out under the action of the reverse coil electromagnetic forces. The electrical energy consumed by the reverse coil from the network is spent on the head kinetic energy change when it is accelerating.

The reverse coil energy balance equation is the following:

$$
\int_{0}^{t_{2}} u_{2} i_{2}-i_{2}^{2} R_{2} d t+\frac{m v_{0}^{2}}{2}=\int_{0}^{v_{2}} m v d v+\Delta w_{\mathrm{m} 2} x, t,
$$

where $u_{2} i_{2}$ is the source power ; $u_{2}, i_{2}$ are correspondingly the current and the resistance of the reverse coil; $\frac{m v_{0}^{2}}{2}$ is the stored head kinetic energy when it is rebounded from the operating instrument; $m, v_{0}$ are correspondingly the mass and initial speed of the head; $v_{2}$ is the head speed in the time point $t_{2} ; \Delta w_{\mathrm{m} 2} x, t$ is the energy of the reverse coil magnetic field. 
Component $\int_{0}^{v_{2}} m v d v$ of the right side of the balance equation determines the head kinetic energy at the end of the interval, which is

$$
\int_{0}^{v_{2}} m v d v=\frac{m v_{0}^{2}}{2}+\int_{0}^{t_{2}} f_{\mathrm{em} 2} v d t-\int_{0}^{t_{1}} f_{\mathrm{em} 1} v d t
$$

where $\int_{0}^{t_{1}} f_{\mathrm{em} 1} v d t$ is the electromagnetic forces work during the head braking by the reverse coil; $\int_{0}^{t_{2}} f_{\mathrm{em} 2} v d t$ is the electromagnetic forces work of the reverse coil.

The head kinetic energy at time point $t_{2}$ is

$$
\int_{0}^{v_{2}} m v d v=\frac{m v_{2}^{2}}{2}
$$

At the time point $t_{2}$ negative voltage half-wave applies to the reverse coil.

The time interval $t_{2} \ldots t_{3}$ is characterized by the head electromagnetic braking.

Mechanical work exceeding the electromagnetic head braking work is converted into magnetic energy with subsequent conversion into electric energy and returning to the network, while compensating the coil heat loss energy

$$
\Delta w_{\mathrm{m} 2} x, t+\int_{t_{2}}^{t_{3}} f_{\mathrm{em} 2} v d t=\int_{t_{2}}^{t_{3}} i_{2}^{2} R_{2} d t+\Delta w_{\mathrm{el} .2} \quad x, t
$$

where $\int_{t_{2}}^{t_{3}} f_{\text {em.c2 }} v d t$ is the mechanical work to overcome the electromagnetic head breaking work; $\Delta w_{\text {el.c2 }} x, t$ is the electrical energy recovered to the network.

It is assumed in the equation mentioned above energy part $\Delta w_{\mathrm{m} 2} \quad x, t$ of the magnetic energy stored by the system in the previous interval of energy transformation could also be spent on compensation of the coil heat loss or partially converted into electric energy and recovered back to the network.

At point $a$ and time $t_{3}$ (Fig. 2), the head reverses and head speed changes into the opposite one.

During the reverse, the energy exchange occurs between the head and moves towards the inertia converter with a greater mass.

At the time of energy exchange, the inertial converter also changes its speed into an opposite sign and its kinetic energy fully transforms into potential energy of compression damper springs:

$$
\frac{m_{\text {in }} v_{\text {in }}^{2}}{2}=\int_{x_{1}}^{x_{2}} k x d x
$$

where $k$ is the spring stiffness coefficient; $k x$ is the compression force of springs; $m_{\text {in }}, v_{\text {in }}$ are correspondingly the inertial converter weight and its initial speed at the moment of impact.

In the time interval $t_{3} \ldots t_{4}$ (Fig. 2), the head movement is carried out only under the action of reverse coil electromagnetic forces in the direction of the operating instrument.

The electrical energy, consumed from the network by the reverse coil, is spent on the head kinetic energy change.

The energy balance equation is:

$$
\int_{t_{3}}^{t_{4}} u_{2} i_{2}-i_{2}^{2} R_{2} d t+\frac{m v_{0}^{2}}{2}=\int_{v_{3}}^{v_{4}} m v d v+\Delta w_{\mathrm{m} 2} \quad x, t
$$

where $\frac{m v_{0}^{2}}{2}$ is the head kinetic energy during the energy exchange with the inertial converter.

The total kinetic energy of the head at the end of the time interval at time point $t_{4}$ is

$$
\int_{v_{3}}^{v_{4}} m v d v=\frac{m v_{0}^{2}}{2}+\int_{t_{3}}^{t_{4}} f_{\mathrm{em} 2} v d t
$$

where $\int_{t_{3}}^{t_{4}} f_{\mathrm{em} 2} v d t$ is the work of reverse coil electromagnetic forces on altering the head kinetic energy.

Time interval $t_{4} \ldots t_{5}$ is characterized by the head electromagnetic braking.

In this time interval, the reverse coil does not consume energy.

Due to head electromagnetic braking external forces, mechanical work is converted into magnetic energy followed by its conversion into electric energy and returning to the network:

$$
\Delta w_{\mathrm{m} 2} x, t+\int_{t_{4}}^{t_{5}} f_{\mathrm{em} 2} v d t=\int_{t_{4}}^{t_{5}} i_{2}^{2} R_{2} d t+\Delta w_{\mathrm{el} .2} x, t
$$

where $\int_{t_{4}}^{t_{5}} f_{\mathrm{em} 2} v d t$ is the work of the external forces during the head electromagnetic braking. 
At time interval $t_{4} \ldots t_{6}$ the voltage impulse applies to the operating coil.

The electrical energy consumed by the operating coil from the network is spent on the head moving in the direction of the working tool kinetic energy changing. This energy is also spent on the head electromagnetic braking by the reverse coil in the period of time $t_{4} \ldots t_{5}$ :

$$
\int_{t_{4}}^{t_{6}} u_{1} i_{1}-i_{1}^{2} R_{1} d t=\int_{v_{4}}^{v_{6}} m v d v+\int_{t_{4}}^{t_{5}} f_{\mathrm{em} 2} v d t+\Delta w_{\mathrm{m} 1} \quad x, t
$$

At the time $t_{6}$ the head impacts the operating instrument. The head kinetic energy for a full cycle will be determined by the total work of the electromagnetic force of the reverse movement that satisfies the energy balance:

$$
\int_{v_{3}}^{v_{4}} m v d v+\int_{v_{4}}^{v_{6}} m v d v=\frac{m v_{\mathrm{st}}^{2}}{2}
$$

where $\frac{m v_{\mathrm{st}}^{2}}{2}$ is the head kinetic energy of the complete cycle; $v_{\text {st }}$ is the head speed before impact.

Thus, the impact energy increase in the double-coil synchronous electromagnetic machine circuit with the head inertial reverse (Fig. 1) is achieved by the cycle time increase and the head impact frequency decrease.

The algorithm of the electromagnetic motor control method includes applying voltages half-waves to the three sequential coils.

Unlike the known controlling methods, the considered one includes the first two half-waves which are applied to the reverse coil, and the third half-wave is applied to the operating coil with noted half-wave voltages sequence without a pause between them.

If this control method is used, the reverse coil provides impact mass acceleration in two directions, and operating coil - in one direction only.

In comparison with the already known control methods, the double-coil synchronous electromagnetic machine circuit with the head inertial reverse allows one to increase the head energy impact with the same values of currents, reducing the effect of the electric drive work on the network.

\section{METHODS OF SOLUTION}

With respect to the generalized forces, established in the system, the mechanical part of the electromagnetic impact machine (Fig. 1) has traditional degrees of freedom of mass inertial links. The mechanical part contains linkages with elastic bonds excited by external electromagnetic forces.

The generalized coordinates include linear movements $x_{1}$ of head mass center $m_{1}, x_{2}$ of tool $m_{2}, x_{3}$ of inertial converter $m_{3}, x_{4}$ of electric drive $m_{4}, x_{5}$ of the fastener with linked mass $m_{5}$.

The spatial oscillations of the system are described by the dependence of the generalized coordinates on time.

The motion equations of the mechanical system are derived from Lagrange equations of the second type:

$$
\frac{d}{d t}\left[\frac{\partial T}{d \dot{x}_{i}}\right]-\frac{\partial T}{d x_{i}}=-\frac{\partial \Pi}{d x_{i}}-\frac{\partial \Phi}{d \dot{x}_{i}}+Q_{i}, \quad i=1,2, \ldots, N,
$$

where $T$ is the system kinetic energy; $\Pi$ is the system potential energy; $\Phi$ is the dissipative function or Rayleigh function; $\frac{\partial \Pi}{d \dot{x}_{i}}, \frac{\partial \Phi}{d \dot{x}_{i}}, Q_{i}$ is the generalized forces of elasticity, resistance to movement and external influences, corresponding to the $x_{i}$-th generalized coordinate; $\dot{x}_{i}$ is the generalized velocity; $N$ is the number of the mechanical system freedom degrees $(N=5)$.

When mass motion in the selected coordinates system is considered, the mechanical system static balance position is assumed as the reference point where the potential energy level is zero.

The mechanical system and the magnetic system are linked by the functions $f_{\mathrm{em} 1}=f i_{1}, x_{1}$ and $f_{\mathrm{em} 2}=f i_{2}, x_{1}$, where $f_{\mathrm{em} 1}, f_{\mathrm{em} 2}$ are the electromagnetic forces, $i_{1}, i_{2}$ are the currents in the inductors, $x_{1}$ is the head position coordinate.

In this case the mechanical and magnetic systems are connected by the dependencies of electromagnetic force $f_{\mathrm{em} 1}=f \quad i_{1}, x_{1}$ and $f_{\mathrm{em} 2}=f \quad i_{2}, x_{1}$.

The magnetic system and the electric system are linked by the functions $\psi_{1}=f i_{1}, x_{1}$ and $\psi_{2}=f i_{2}, x_{1}$, where $\psi_{1}$, $\psi_{2}$ are flux linkages.

The electric balance equations have the form:

$$
\begin{aligned}
& u_{1} t=i_{1} r_{1}+\frac{d \psi_{1} i_{1}, x_{1}}{d t}, \\
& u_{2} t=i_{2} r_{2}+\frac{d \psi_{2} i_{2}, x_{1}}{d t},
\end{aligned}
$$

where $u_{1} t, u_{2} t$ are the windings voltages; $r_{1}, r_{2}$ are the pure resistances of inductors circuits.

The real motion process should be considered as the result of superposition of forced oscillation, free oscillation and periodic impact pulses of the forces in the mechanical system with respect to power loss.

The reciprocated head motion is: 


$$
x_{1} t=\left\{\begin{array}{l}
h_{2}-h_{1}-\delta_{2}^{+}, 0 \leq x_{1} \leq h_{2}-h_{1} ; \\
h_{2}-h_{1}+\delta_{2}^{-}, x_{1}>h_{2}-h_{1},
\end{array}\right.
$$

where $h_{1}, h_{2}$ are the fixed coordinates relative to the impact section size of the working tool; $\delta_{2}^{+}, \delta_{2}^{-}$are the variable values of the air working gap formed by the position of the striker relative to the upper and lower pole system of the return coil.

Solution of the differential equations system (1)-(3) with respect to (4) is aimed to describe the dynamics of electromagnetic impact unit.

The obtained equations permit to make the detailed analysis of the operating processes with respect to nonlinearities in the magnetic system, the degree of inertial masses mobility and properties of mechanical system spring linkages with respect to impact interactions and power loss.

\section{Simulation RESUltS}

The dynamical model implementation is based on the arrays of reference points of static parameters of the flux linkages $\psi_{1}=f i_{1}, x_{1}, \psi_{2}=f i_{2}, x_{1}$ and electromagnetic forces $f_{\mathrm{em} 1}=f i_{1}, x_{1}, f_{\mathrm{em} 2}=f i_{2}, x_{1}$ with respect to inductors currents and head position. The static parameters can be found with FEMM or ELCUT.

The differential equations system was solved with the structured modeling tool in Matlab Simulink.

Fig. 3 and Fig. 4 state the simulation results as time diagrams of the switching-on process of the electromagnetic impact unit with controllable impact energy up to $6 \mathrm{~J}$ and shocks synchronous frequency $n_{\mathrm{sh}}=2000 \mathrm{~min}^{-1}$.

The initial data for simulation are taken from the electromagnetic impact unit IE 4207.

The model power voltage was taken from the single-phase source with amplitude $311 \mathrm{~V}$ and frequency $50 \mathrm{~Hz}$. The periodic current pulses were generated by controllable gates with a wide control range.

The power loss current components were taken into account according to the mentioned above recommendations.

The proposed duty cycle was compared with the known one, implemented in the two-inductor synchronous electromagnetic machine with inertial head reverse.
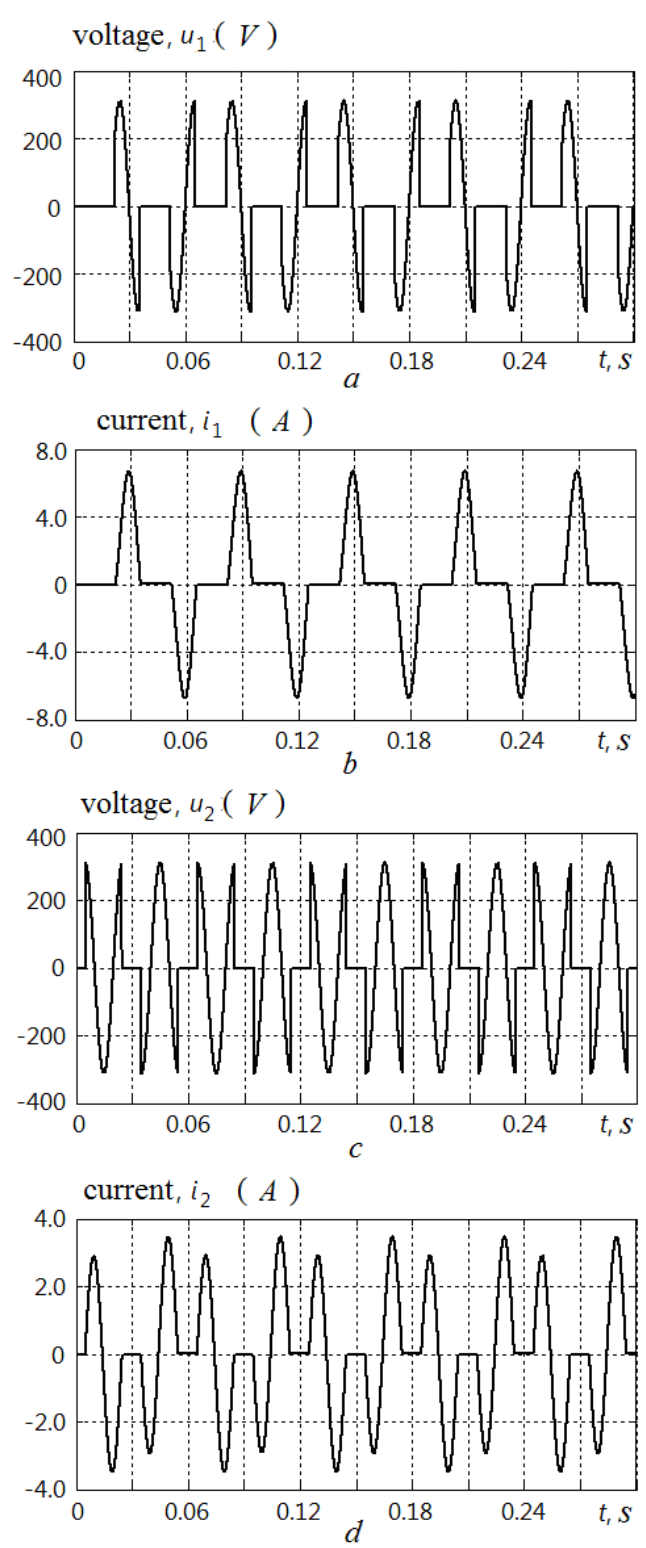

Fig. 3. Time diagrams of the electromagnetic impact node switchingon process: $\mathrm{a}$ - travel inductor voltage; $\mathrm{b}$ - reverse induction voltage; $\mathrm{c}$ - inductor travel current; $\mathrm{d}$ - reverse inductor current

The preliminary simulation results comparison shows that the combined duty cycle together with the control method provides the current amplitude decrease up to $30 \%$ and it reduces the electric drive influence on the power line. 

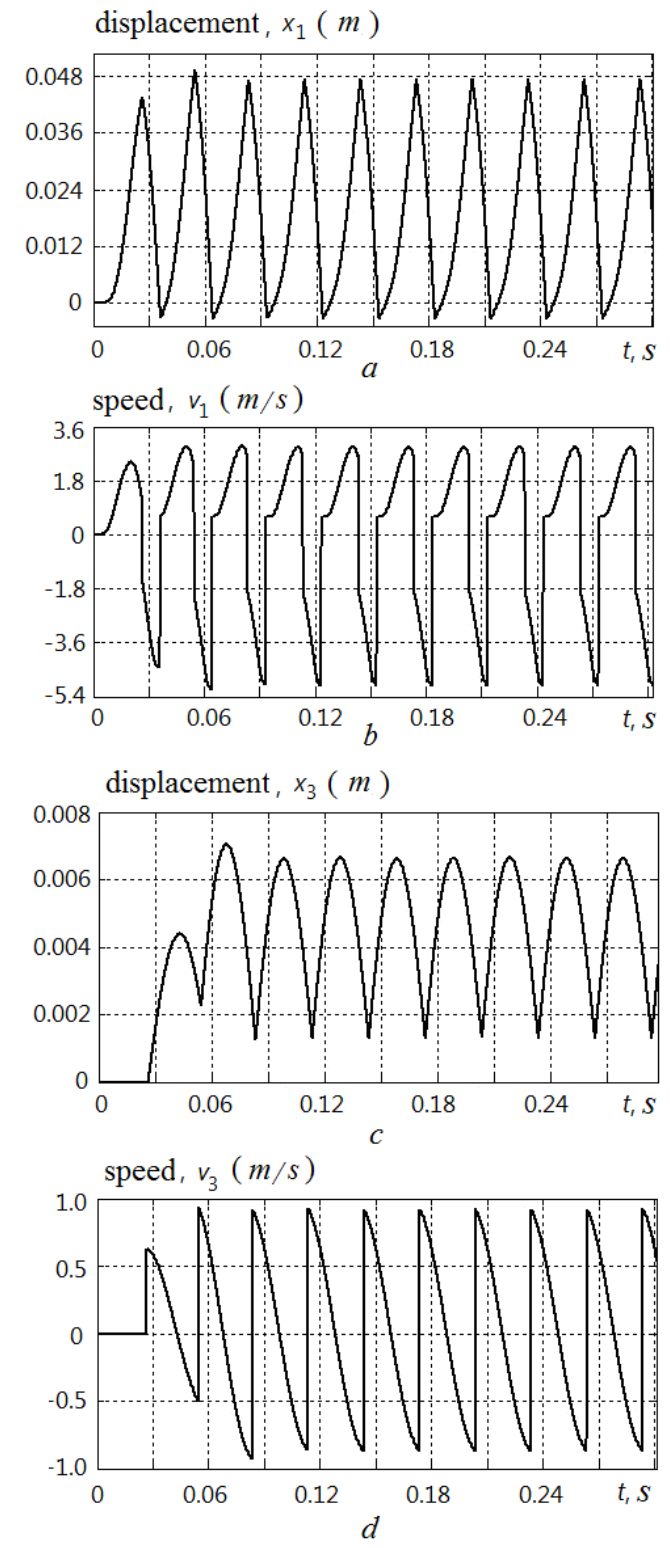

Fig. 4. Time diagrams of the process of switching on the electromagnetic impact unit: $a$ - displacement of the striker; $b$ - is the speed of the striker; $\mathrm{c}-$ displacement of the inertial converter; $\mathrm{d}-$ is the speed of the inertial converter.

\section{RESULTS AND CONCLUSION}

In comparison with a known operation system, the implementation of a new operation cycle in a linear synchronous double-coil electromagnetic machine with the inertial head reverse allows increasing the single impact energy without increasing the effect of the electric drive work on the network.

The considered algorithm and control method in the double-coil electromagnetic machine with an inertial head reverse schema allows using three voltage half-wave instead of two during the operation cycle as in other well-known controlling methods.

The dynamical model of the two-inductor synchronous impact electromagnetic machine with inertial head reverse was developed. This model gives wide capabilities of the detailed analysis of operating processes in transient and quasistationary modes when periodic impact force pulses are generated and interact with deformed medium.

The combined duty cycle provides less current amplitude and improved electromagnetic compatibility in comparison with the known one when the impact node is powered from the single-phase $50 \mathrm{~Hz}$ power source.

\section{REFERENCES}

[1] N.P. Ryashentsev, V.N. Ryashentsev, Electromagnetic drive of linear machines, Novosibirsk: Science, 1985.

[2] V.P. Pevchev, "The superexitation and efficiency relation in a shortstroke pulsed electromagnetic motor of a seismic source", Journal of Mining Science, vol. 46, pp. 656-665, June, 2010.

[3] S.N. Kuchankov, E.M. Timoshenko, "Nonsteady thermal conditions of electromagnetic motors in shock-type equipment", Journal of Mining Science, vol. 34, pp. 148-152, February, 1998.

[4] L.A. Neiman, V.Y. Neiman, "Linear synchronous electromagnetic machines for lowfrequency impact technologies", Russian Electrical Engineering, vol. 85, pp. 752-756, December 2014.

[5] V.Y. Neiman, L.A. Neiman, A.A. Petrova, "Calculation of efficiency of DC electromagnet for mechanotronbic systems", IFOST-2008. Novosibirsk-Tomsk, Russia, pp. 452-454, June 2008. [Proc. of the 3d International Forum on Strategic Technology, Novosibirsk-Tomsk, Russia, 2008]. 